\title{
Distance Measurement in Middle Ear Surgery Using a Telemanipulator
}

\author{
Thomas Maier $^{1}$, Gero Strauss ${ }^{2}$, Franz Bauer ${ }^{1}$, \\ Andreas Grasser ${ }^{1}$, Nobuhiko Hata ${ }^{3}$, and Tim C. Lueth ${ }^{1}$ \\ ${ }^{1}$ Institute of Microtechnology and Medical Device \\ Technology (MiMed), Technische Universität München, Germany \\ thomas.maier@tum. de \\ ${ }^{2}$ Department of ENT, University Hospital Leipzig and BMBF Innovation \\ Center Computer Assisted Surgery (ICCAS), University Leipzig, Germany \\ gero.strauss@medizin.uni-leipzig.de \\ ${ }^{3}$ Department of Radiology, Harvard Medical School, Brigham and Women's Hospital, USA \\ hata@bwh. harvard.edu
}

\begin{abstract}
In this article, a new tool for the intraoperative measurement of distances within the middle ear by means of a micromanipulator is presented. The purpose of this work was to offer the surgeon a highly accurate tool for measuring the distances between two points in the 3D operational field. The tool can be useful in various operations; this article focuses, however, on measuring the distance between the stapes footplate and the long process of the incus of the middle ear. This distance is important for estimating the proper prosthesis length in stapedotomy for treating otosclerosis. We evaluated the system using a simplified mechanical model. Our results show that the system can measure distances with a maximum error of $0.04 \mathrm{~mm}$.
\end{abstract}

Keywords: Middle Ear Surgery, Telemanipulator, Stapedotomy, Measurement.

\section{Introduction}

The intraoperative measuring of anatomical structures is necessary in many surgical interventions, in order to evaluate preoperative situations and postoperative results. With a large surgical access, this can be done easily. In the small operational field of Middle Ear Surgery, however, this is much more difficult [1]. An example is the measurement of the distance between the long process of the incus and stapes footplate in a stapedotomy. Stapedotomy is a surgical technique for treating conductive hearing loss owing because of ossification of the oval window. Through stapedotomy, the stapes is replaced by a small prosthesis (Fig. $1 \mathrm{a}, \mathrm{b}$ ). The prosthesis should protrude about $0.2-0.3 \mathrm{~mm}$ into the inner ear [2]. Depending on the patient's anatomy, prostheses of different length (e.g., $3.25 \mathrm{~mm}-4.7 \mathrm{~mm}$ ) are used.

The prosthesis length has a great impact on the hearing results. A prosthesis that is too long can lead to dizziness, sudden pressure gains, and conductive hearing loss. A prosthesis that is too short, however, can lead to a displacement of the prosthesis. Furthermore, the perforation in the stapes footplate can be overgrown, which 
subsequently leads to an interception of sound conduction. Results can be conductive hearing loss and perilymph fistula [4, 2]. A revision surgery, in which the incorrect prosthesis is replaced by a suitable one, can be necessary. According to literature, 7$28 \%$ of the stapes revision surgeries can be directly traced back to incorrect prosthesis length [4, 3]. Gros et al. even indicates that incorrect prosthesis length is a major reason for prosthesis dislocations, which are the cause of $18-82 \%$ of the revisions [4].

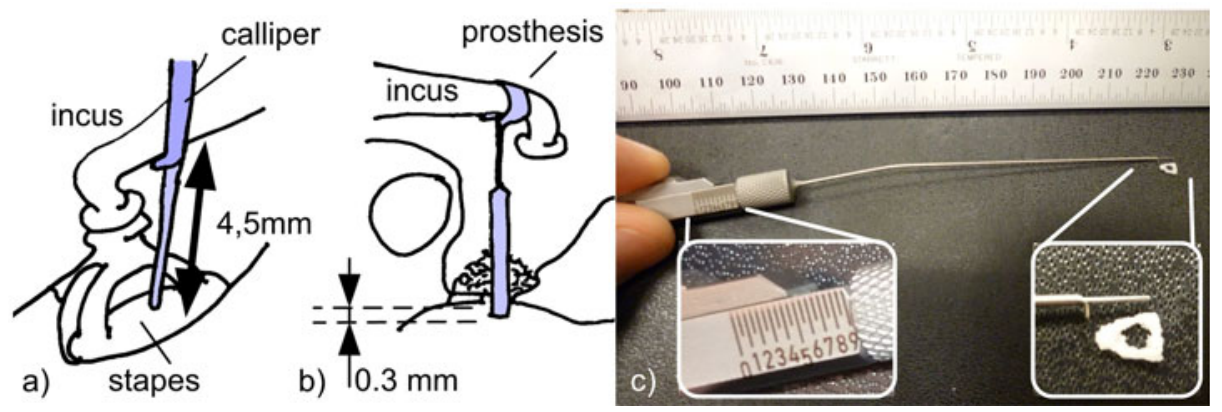

Fig. 1. a) Distance between incus and stapes footplate. b) Stapes replaced by prosthesis. Calliper with stapes model (Heinz Kurz GmbH Medizintechnik, Dusslingen, Germany).

In clinical practice, intraoperative measurements of anatomical structures during middle ear surgeries are performed with special callipers (Fig. 1a, c), measuring rods, sizers or through estimation based on anatomical landmarks [1]. Most often, a prosthesis length of $4.5 \mathrm{~mm}$ is required. Hence, some surgeons use only this single prosthesis type or try to estimate the proper length. Kwok et al. assume that it is impossible to do an accurate estimation of the distance between the long process of the incus and the stapes footplate without measuring devices, owing to the angle of view. Furthermore, Strauss et al [1] describe the magnification and the low depth of field caused by the surgical microscope as difficult for estimations. In [5], optical coherence tomography (OCT) is used to determine the proper prosthesis length. Moreover, the OCT measurements are compared with those performed with a mechanical calliper. It is found that measuring with a calliper leads to an error of up to $0.4 \mathrm{~mm}$. In research, the positioning of prostheses was analyzed postoperatively with high-resolution CT in [6]. It was found that HR-CT data is not suitable for doing accurate measurements in this scale. In [1], a new optical multipoint lasermeasurement system (KARL STORZ GmbH \& Co. KG, Tuttlingen, Germany) for a flexible endoscope is introduced for ENT-surgery. The accuracy is reported to be 0.2 to $0.5 \mathrm{~mm}$.

The distance measurement between stapes footplate and long process of the incus during stapedotomy involves strict requirements in terms of the accuracy and usability of the measuring system. Hence, this measurement was chosen representatively for many other measurement tasks in ENT-Surgery. The system should provide measurements with accuracy better than $0.1 \mathrm{~mm}$ within the operational field. We propose a telemanipulated measurement system with 3 degrees of freedom (DOF) and highly accurate position sensors. By touching two anatomical landmarks using arbitrary microinstruments like perforators or forceps, their relative distance should be 
calculated and visualized. Measurements should be able to be taken in stand-alone operation of the manipulator or in combination with external devices, such as navigation systems or systems for image processing.

\section{Description of the Measuring System}

The measuring system used consists of a teleoperated micromanipulator with joystick control, three highly accurate position sensors, an independent microcontroller-based evaluation electronics, a visualization software, and a bridge software for protocol transformation (Fig. 2).

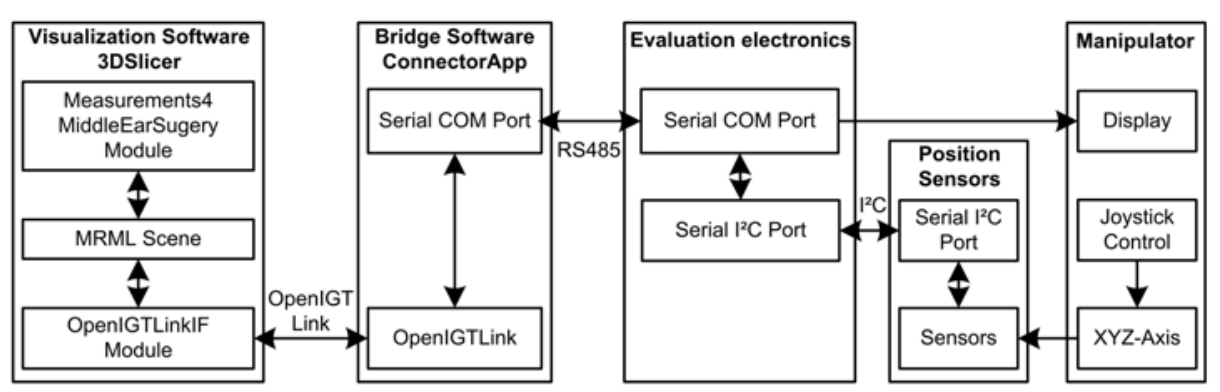

Fig. 2. Block diagram of the overall measuring system

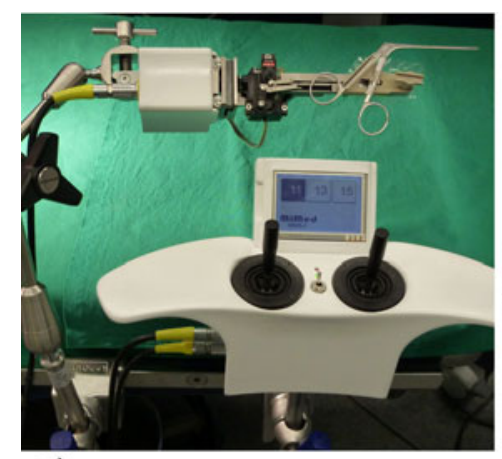

a)

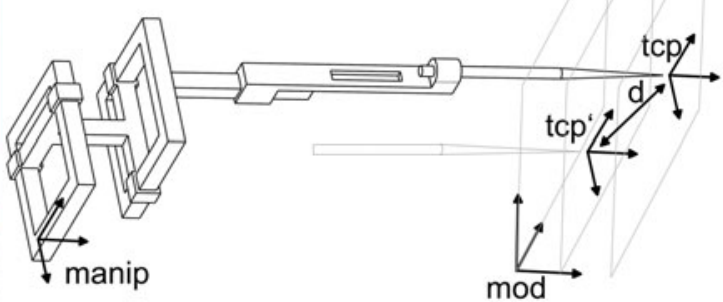

b)

Fig. 3. a) Micromanipulator System with joystick control. b) Cartesian kinematics of the Manipulator with 3DOF. The distance between two tcp-positions can be calculated within the CT model.

Micromanipulator: A Micromanipulator System developed for middle ear surgery was introduced in [7] (Fig. 3). This system is based on previous experience with large ceiling-mounted interactive robots [8] and small autoclavable robots [9-10]. We used this manipulator as the basis for our measuring system. It is small-sized, lightweight, and capable of moving standard instruments of ENT surgery with high accuracy in a 
small workspace. It can be already be integrated into standard operating procedures and allows rapid change between manual and teleoperated instrument guidance. The micromanipulator has three translational DOF in Cartesian configuration. An additional DOF is used to open and close attached forceps. The MMS-II is mounted on the OR table's side rail and aligned with the operating field, using an articulated arm. The joystick controller is mounted on the OR table's side rail directly in front of the surgeon.

Measuring System and Sensors: Each axis of the manipulator was equipped with an additional position sensor. Because of their high precision, small overall size, and simple interface, hall-sensors of the type TRK-1T02 Tracker (New Scale Technologies Inc., Victor, NY, USA) were chosen. They are able to detect an absolute position within a terminal pair $(2 \mathrm{~mm})$ with 12-bit resolution. By detecting the crossover from one terminal pair to the next, we are able to cover an arbitrary measuring section. Positions can be read out via $\mathrm{I}^{2} \mathrm{C}$ serial bus. The strength and gradient of the magnetic input are analyzed in order to check the integrity of the data obtained. According to the manufacturer, the maximum absolute error is $\pm 10 \mu \mathrm{m}$.

Evaluation Electronics: A microcontroller-based evaluation electronics was built to readout the sensors. An Atmega 168 microcontroller (Atmel Corporation, San Jose, CA, USA) with $20 \mathrm{MHz}$ and 8 bit is used as a central processing unit. The controller features an $\mathrm{I}^{2} \mathrm{C}$ interface for communicating with the position sensors. Furthermore, the evaluation electronics has an interface for sending the current position continuously $(20 \mathrm{~Hz})$ to external components and devices via RS232 or RS485. This is used to visualize the manipulator at an external navigation system. Additionally, the current position can be sent and saved as a measuring value. Therefore, the sensor readings are averaged within a time interval of 0.5 seconds to increase accuracy. The averaged position is sent and stored as a measuring value only when the sensor readings have been stable in that time interval. The communication protocol used is proprietary to our lab and enables communication with other robotic and imageprocessing devices. This ensures modularity. The Euclidian distance between two measuring values is also calculated directly by the microcontroller in order to be displayed at the joystick control, if stand-alone operation without navigation is needed.

Visualization Software: To visualize the measuring values and results, the opensource software 3DSlicer (www.slicer.org) was used. 3DSlicer is a cross-platform application for visualizing and analyzing medical-image data. With the help of various modules, image and sensor data can be recorded, processed, and displayed. Various data (images, positions, transforms, etc.) can be exchanged with external devices using the OpenIGTLink protocol [11]. We used the data type TRANSFORM to exchange data with 3DSlicer. The evaluation electronics provides the position data and measurement values using our laboratory's own proprietary protocol, which is not compatible to OpenIGTLink. Therefore, a bridge software (ConnectorApp) was implemented. This software transforms message data received from the proprietary protocol to the OpenIGTLink protocol. The ConnectorApp has the additional task of coordinating the different working steps (initialization, registration, and measurement). It further transforms positions from manipulator coordinates to 3DSlicer image coordinates. The calculation of the distance between two measuring values and the 
visualization of those points and their distance are carried out by the newly developed "Measurements4MiddleEarSurgery" (MMES) module for 3DSlicer.

Initialization and Measuring Process: The system usage introduced is divided into three steps: initialization, registration, and measurement. The initialization step sets up communication between 3DSlicer, ConnectorApp, and evaluation electronics. For that purpose, the 3DSlicer module "OpenIGTLinkIF" is used. After that, basic information is interchanged. This is necessary to define the way data is exchanged in the next steps. During the registration step, the transformation matrix between 3DSlicer's manipulator coordinates (manip) and image coordinates (mod) must be calculated. Therefore, three landmarks are set in the image dataset loaded within 3DSlicer. This is done by means of the "Fiducials" module. Possible landmarks are bony structures within the middle ear, e.g. the bony canal of the facial nerve. After that, the three points are sent to the ConnectorApp using OpenIGTLink. The same landmarks are then set with the manipulator's tooltip and sent to the ConnectorApp, which is then able to calculate the necessary transformation matrix. This step can be skipped if the visualization of points within the 3D image dataset is not required. Finally, the third step--the measurement--can be started. The evaluation electronics starts transmitting the current position every $50 \mathrm{~ms}$. The ConnectorApp performs the coordinate transformation and routes the data via OpenIGTLink to 3DSlicer. 3DSlicer receives the data within the OpenIGTLinkIF module. The position of the visualized tool tip can now be updated. To record a measurement value, a command can be sent to the evaluation electronics by a simple switch or using the serial port. As described above, the sensor readings are averaged; their correctness is tested; and the resulting measurement value is sent to 3DSlicer. As soon as a second measurement value is received by 3 DSlicer, the distance between those two is calculated and visualized.

\section{Experiments and Results}

Two Experiments were conducted to validate the measuring system. In the first experiment, the accuracy itself of the sensor system was determined. In the second experiment, the telemanipulated instrument guidance was considered in the accuracy measurement.

\section{Experiment 1: Accuracy of the Sensor System}

In this experiment, the accuracy of the sensor system was valuated. Therefore, the thickness of two precise gauge blocks was determined. The manipulator motors were deactivated, so that the manipulator's precision would not affect the measurement results. Five measuring points were marked on a measuring table within an area of 15 $\mathrm{x} 15 \mathrm{~mm}^{2}$. The Manipulator was positioned in such a way that the axis of the inserted instrument (STORZ Perforator, $0.3 \mathrm{~mm}$ ) was oriented perpendicular to the measuring table. The instrument tip was moved manually toward the first measuring point. A first measurement value was recorded at that position. After that, the instrument tip was moved upward. Two precision gauge blocks, with a thickness of $2 \mathrm{~mm}( \pm 0.01$ $\mathrm{mm})$ and $5 \mathrm{~mm}( \pm 0.01 \mathrm{~mm})$ respectively, were put onto the measuring table. Then, the instrument tip was set down again, and this position was stored as the second 
measuring value. The calculated distances, shown by 3DSlicer, were stored. This procedure was repeated twenty times for each of the five measuring points. The mean and the standard deviation (SD) are shown in Table 1.

Table 1. At five measuring points, the thickness of two gauge blocks was determined

\begin{tabular}{ccccccc}
\hline & Measuring Point & $\mathbf{1}$ & $\mathbf{2}$ & $\mathbf{3}$ & $\mathbf{4}$ & $\mathbf{5}$ \\
\hline \multirow{2}{*}{ gauge block: } & mean/mm (n=20) & 2.00 & 2.00 & 2.00 & 2.00 & 2.00 \\
$2.00 \mathrm{~mm} \pm 0.01 \mathrm{~mm}$ & $\begin{array}{c}\text { SD/mm } \\
\text { maximum error }\end{array}$ & 0.01 & 0.01 & 0.01 & 0.01 & 0.01 \\
& mean/mm (n=20) & 5.00 & 4.99 & 4.99 & 5.01 & 5.00 \\
\hline gauge block: & $\mathbf{S D} / \mathbf{m m}$ & 0.01 & 0.01 & 0.01 & 0.01 & 0.01 \\
& maximum error & & & 0.02 & & \\
\hline
\end{tabular}

\section{Experiment 2: Accuracy of the telemanipulated measuring system}

In the second experiment, the entire measuring system, including the telemanipulated positioning of the tool tip, was valuated. A phantom was developed to imitate the task of measuring the distance between incus and stapes footplate (Fig. 4). It consists of a rod $(\varnothing 1 \mathrm{~mm})$ fastened to a cylinder by means of two elastic bands. This imitates the movable incus. The cylinder has an inner diameter of $7 \mathrm{~mm}$, representing the ear canal. In the initial position, the distance between the rod and the bottom of the cylinder is $4.30 \mathrm{~mm}( \pm 10 \mu \mathrm{m})$. A $1.5-\mathrm{mm}$ microhook was clipped onto the manipulator. The instrument and the phantom were connected with simple electronics signaling the physical contact between both, by means of an LED. This was done in order to eliminate operator and visual-condition influence.

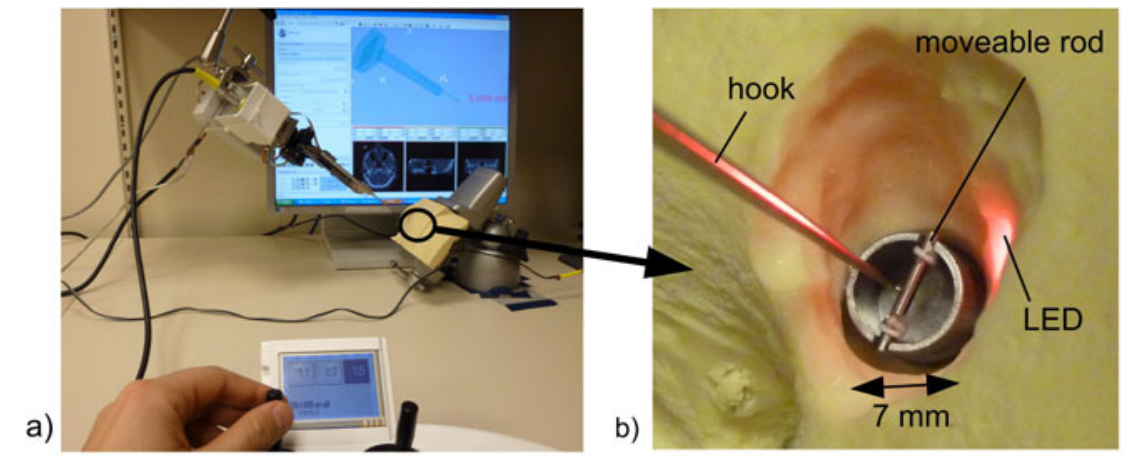

Fig. 4. a) Experimental setup. b) Detailed view of the middle-ear phantom.

The Manipulator was oriented in a way that allowed the instrument axis to be perpendicular to the cylinder ground. The instrument was moved toward the bottom (stapes footplate), using the joystick control, until the LED lighted up. The measuring value was sent to 3DSlicer. Then, the hook was moved toward the bottom side of the 
rod (proximal side of the incus) until the LED lighted up. This measuring value was sent to 3DSlicer as well. The measuring result displayed by 3DSlicer was stored. Because of the moveable rod, the positioning errors of the manipulator system are included in the measuring result, in contrast to the first experiment. The results are shown in Table 2. The $0.30-\mathrm{mm}$ thickness of the hook itself has to be added to the measuring value.

Table 2. Results for telemanipulated measuring

\begin{tabular}{ccc}
\hline reference value: & mean $/ \mathbf{m m}(\mathbf{n}=\mathbf{5 0})$ & $4.01 \mathrm{~mm}(+0.30 \mathrm{~mm}$ hook $)$ \\
$4.30 \mathrm{~mm} \pm 0.01 \mathrm{~mm}$ & $\mathbf{S D} / \mathbf{m m}$ & $0.02 \mathrm{~mm}$ \\
& maximum error & $0.04 \mathrm{~mm}$ \\
\hline
\end{tabular}

Results: The first experiment shows that the position sensors can determine the tooltip position with a precision (two standard deviations) of $\pm 0.02 \mathrm{~mm}$. The small deviation of the mean value shows that there is no systematic error. The second experiment shows that the manipulator is able to touch a surface so precisely that the high accuracy of the sensors can be used in practical applications.

\section{Conclusion}

We have developed a measuring system based on a miniature telemanipulator for precise determination of distances within a small workspace. The aim of our work was to increase measuring accuracy in middle ear surgery. This article was focused on one specific task representing many others: the estimation of the distance between the stapes footplate and the long process of the incus during a stapedotomy. An available micromanipulator with 3DOF was equipped with an independent, highly accurate position-sensor system. The open-source software 3DSlicer (www.slicer.org) was used for visualizing the measuring results. The measuring system can also be operated without visualization and navigation in a stand-alone mode. Precise measuring is crucial in many surgical procedures. Measurements accurate to a tenth, however - as required in middle-ear surgery - cannot be achieved with common methods. Our solution can provide measurements with a maximum error of $0.04 \mathrm{~mm}$, which was valuated with a technical phantom. Preliminary experiments show, that the contact of a tooltip with the tissue's surface can be determined very precisely $(<0,05 \mathrm{~mm})$, when using a microscope. Nevertheless, this in vivo precision has to be determined in further experiments with a larger group of surgeons, because it could depend on individual skills.

Acknowledgement. The authors wish to thank Sam Song and Junichi Tokuda for their support. We also wish to thank KARL STORZ and Heinz KURZ for supporting us with medical instruments. This work has been supported by the German Research Foundation (DFG, PAK 404) and several NIH funds (5U41RR019703, 5P01CA067165, 1R01CA138586, 1R01CA124377, 2R44DE019322). 


\section{References}

1. Strauss, G., Hipp, S., Fischer, M., Hofer, M., Munz, F., Dietz, A., Strauss, M., Lüth, T.: Intraoperative volumetry in ENT surgery. Objectivation of Surgical Success by the Volume Control System. HNO 58, 839-845 (2010)

2. Theissing, J., Rettinger, G., Werner, J.A.: ENT-Head and Neck Surgery: Essential Procedures. Thieme Medical Pub. (2010)

3. Edelstein, D.R.: Revision Stapedectomy and Stapedotomy. Thieme (2008)

4. Gros, A., Vatovec, J., Žargi, M., Jenko, K.: Success rate in revision stapes surgery for otosclerosis. Otology \& Neurotology 26, 1143-1148 (2005)

5. Heermann, R., Hauger, C., Issing, P.R., Lenarz, T.: Application of Optical Coherence Tomography (OCT) in Middle Ear Surgery. Laryngo-Rhino-Otologie 81(6), 400-405 (2002)

6. Bozzato, A., Struffert, T., Hertel, V., Iro, H., Hornung, J.: Analysis of the accuracy of highresolution computed tomography techniques for the measurement of stapes prostheses. European Radiology 20, 566-571 (2010)

7. Maier, T., Strauss, G., Hofer, M., Kraus, T., Runge, A., Stenzel, R., Berger, T., Dietz, A., Lueth, T.C.: A New Micromanipulator System for Middle Ear Surgery. In: IEEE International Conference on Robotics and Automation, pp. 1568-1573 (2010)

8. Hein, A., Lueth, T.C.: Image-Based Control of Interactive Robotics Systems. In: Taylor, C., Colchester, A. (eds.) MICCAI 1999. LNCS, vol. 1679, pp. 1125-1132. Springer, Heidelberg (1999)

9. Schauer, D., Hein, A., Lüth, T.C.: Dynamic force control for a miniaturised medical robot system. In: Proceedings 2003 IEEE/ASME International Conference on Advanced Intelligent Mechatronics (AIM 2003), pp. 1090-1095. IEEE, Port Island (2003)

10. Koulechov, K., Rapoport, T., Lueth, T.C.: Miniaturized, Autoclavable Surgical Robot (Miniaturisierter, autoklavierbarer Roboter für die Chirurgie). at - Automatisierungstechnik 54, 213-221 (2006)

11. Tokuda, J., Fischer, G.S., Papademetris, X., Yaniv, Z., Ibanez, L., Cheng, P., Liu, H., Blevins, J., Arata, J., Golby, A.J., Kapur, T., Pieper, S., Burdette, E.C., Fichtinger, G., Tempany, C.M., Hata, N.: OpenIGTLink: an open network protocol for image-guided therapy environment. The International Journal of Medical Robotics and Computer Assisted Surgery 5, 423-434 (2009) 